\title{
Tangential soft $x$-ray imaging for shape and current profile measurements
}

\author{
K. Tritz, ${ }^{\text {a) }}$ R. Fonck, M. Reinke, and G. Winz \\ University of Wisconsin, Madison, Madison, Wisconsin 53706
}

(Presented on 11 July 2002)

\begin{abstract}
Flux surface shape information is imaged with an x-ray pinhole camera and used as a constraint for reconstruction of the plasma current and $q$ profiles of plasmas in the low-aspect ratio PEGASUS Toroidal Experiment. The camera system is comprised of a $30 \mathrm{~cm} \mathrm{Gd}_{2} \mathrm{O}_{2} \mathrm{~S}$ : $\operatorname{Pr}$ scintillator plate whose $\mathrm{x}$-ray incident side is lens coupled to a microchannel plate (MCP) image intensifier. A slow-scan charge coupled device (CCD) camera is then lens coupled to the MCP for a time resolution of $\sim 1 \mathrm{~ms}$. This system will provide photon noise $<1 \%$ with a $1 \mathrm{~ms}$ integration time for intensities typical of Pegasus plasmas. Installation of a $0.2 \mu \mathrm{m}$ nickel $\mathrm{x}$-ray filter provides necessary contrast between the hot interior and cooler plasma edge region. The scintillator system is compared to direct X-ray illumination of a CCD sensor. Direct illumination uses a custom chip mask and frame transfer for exposure control and sampling at multiple time points. Increased sensitivity of direct illumination imaging system will raise the signal to noise ratio allowing for shorter integration times. (C) 2003 American Institute of Physics. [DOI: 10.1063/1.1537876]
\end{abstract}

\section{INTRODUCTION}

Determination of the current profile is crucial for understanding the stability properties of most toroidally confined plasmas. The plasma current profile can be inferred with measurements of the internal magnetic field structure using diagnostics such as motional Stark effect and Faraday rotation. However, the development of the small aspect ratio toroidal device, with compact geometry and low toroidal field, brings with it many issues that complicate the implementation of these standard diagnostics. Therefore, there is a need to develop and implement a diagnostic that can contribute to the reconstruction of the full plasma magnetic equilibrium and is not hindered by the restrictions of these new devices.

This article discusses the implementation of a tangentially viewing, soft x-ray pinhole camera (SXR PHC) on the Pegasus Toroidal Experiment ${ }^{1}$ for determination of the plasma current profile through imaging of the plasma flux surfaces. Preliminary feasibility modeling of an x-ray imaging system for the Pegasus device was reported in an earlier work. ${ }^{2}$ The measurements from the PHC are used as constraints in an equilibrium reconstruction code just as the measurements from MSE or Faraday rotation would be used. The simplicity of the diagnostic combined with the passive nature of the measurement obviates the need for an expensive neutral beam or complex wave launching and receiving instruments. The SXR PHC system will also scale well as fusion experiments progress to higher temperatures and densities, whereas other profile diagnostics may encounter complications from beam attenuation and frequency cutoff. In addition, the sensitivity of flux surface shape measurements benefit greatly from the extreme shaping found both in low aspect ratio plasmas and also in the advanced tokamak regimes.

The relationship between the shapes of the flux surfaces

${ }^{a)}$ Electronic mail: tritz@cae.wisc.edu and the plasma current profile has been demonstrated theoretically by Christiansen and Taylor, ${ }^{3}$ and observed experimentally on several devices. One method of measuring the shapes of the flux surfaces is to measure the x-ray emission from the plasma. X-ray emission is dependent on the temperature, density, and impurity content of the plasma. If these quantities are assumed to be constant on a flux surface, than the x-ray emission should also be constant on that flux surface. Investigations using tomographic inversion on Alcator demonstrated that the assumption of equal emissivity on plasma flux surfaces may not always hold true. In fact, there is evidence that strong local heating and poloidal flows can lead to asymmetric impurity concentrations, especially in the outer radius of the plasma. ${ }^{4}$ On the JET tokamak, tomographic inversion of many chordal measurements was modestly successful in reconstructing the plasma equilibrium, though sensitivity to noise, inherent in any direct inversion process, prevented the measurements from accurately determining the central safety factor $q_{0} \cdot{ }^{5}$

An earlier version of the tangential PHC was implemented on the Princeton Beta Experiment (PBX) using a forward modeling technique to avoid the difficulties of the direct inversion process. ${ }^{6,7}$ The results of an equilibrium reconstruction code were toroidally projected and compared against the measured two-dimensional (2D) tangential image. The value of $q_{0}$ was manually changed in the reconstruction until the projection and measured image had the closest match. Though the use of the image required a manual iterative feedback loop, this technique demonstrated that the incorporation of flux surface shape information along with the other external magnetic diagnostics as a constraint on the reconstruction was a robust method for determination of the plasma current profile.

The system described in this paper takes the aforementioned technique a step further. The $2 \mathrm{D}$ tangential image is used directly as a constraint in the equilibrium reconstruction code along with other external measurements. The code combines the equilibrium solver, measurement fitting and 


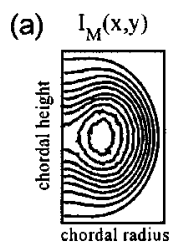

(b)

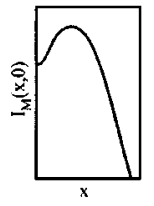

(c)

(d)

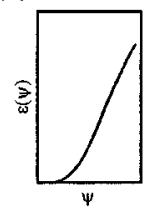

(e) $\psi(\mathrm{R}, \mathrm{Z})$

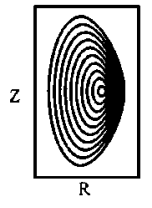

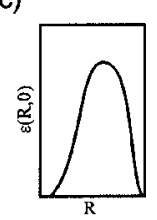

(f) $I_{c}(x, y)$

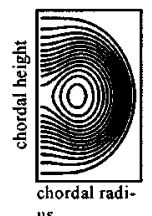

FIG. 1. Technique to constrain equilibrium code using SXR pinhole camera. (a) SXR intensity image from camera, (b) midplane of measured intensity image, (c) Abel inversion of intensity to obtain emissivity profile, (d) emissivity mapped to a flux profile generated from equilibrium code, (e) map used to create model emissivity cross section, and (f) model intensity projection generated to compare with (a).

image projection routines to find the equilibrium that best satisfies all of the measurement constraints.

\section{IMPLEMENTATION}

As is true with all other current profile diagnostics, flux surface shape is not a direct measure of the $q$ and current profiles. Instead, this information must serve as an input constraint to a magnetic equilibrium reconstruction code. The code then uses this constraint, along with external magnetics and other diagnostics, to converge to a solution that best fits the measurement values.

The reconstruction of the PEGASUS Equilibrium uses the Grad-Shafranov PDE solver from Tokamac ${ }^{8}$ (a reconstruction code developed by Mike Mauel, Columbia University) combined with an external Levenberg-Marquardt (LM) nonlinear least squares fitting routine. ${ }^{9}$ Though the nonlinear fitting routine is slower than the usual linear least squares fitting used by other codes, including the original Tokamac code and EFIT, it has the advantage of being robust and extremely flexible. Any property of the equilibrium that can be quantified can be used as a constraint on the reconstruction. The flexibility of the LM fitting algorithm was necessary to simplify the incorporation of the tangential x-ray image as a constraint for the reconstruction. There is no need to parameterize or linearize the $\mathrm{x}$-ray image as the measurement minimization merely depends on the residual of the difference between the model projection and the original image. Additionally, the Grad-Shafranov (GS) solver was modified to include spline parameterizations of the pressure and current profiles. The previous GS core only had a power scaling and polynomial representation of the profiles which were either too restrictive or introduced unrealistic oscillatory structure to the profiles.

The process by which the tangential image is used as a constraint is as follows (Fig. 1). First, the intensity on the vertical midplane of the image is Abel inverted to obtain $\mathrm{x}$-ray emissivity as a function of major radius. Next, an equilibrium reconstruction iteration is obtained from the GradShafranov solver. The midplane flux from the reconstruction combined with the $\mathrm{x}$-ray emissivity provides a mapping of

emissivity to flux. This mapping is then used to generate an emissivity cross-section using the same equilibrium iteration. The cross section is projected to form a simulated tangential projection. This model is compared to the original tangential image. The residual of this comparison is used as an equilibrium constraint which, when minimized, results in a current profile reconstruction that best matches the shape data contained in the $\mathrm{x}$-ray image.

The development of the new nonlinear fitting equilibrium reconstruction code allowed direct modeling of the sensitivity of plasma reconstructions to the shapes of the flux surfaces. The effectiveness of two different constraints was explored with the code; the elongation of the flux surfaces, and a model 2D tangential image.

\section{MODELING}

The elongation of specific flux surfaces proved to be a very strong constraint on the plasma current and $q$ profiles. As the elongation measurement was moved to flux surfaces with higher normalized flux (i.e., further out in plasma minor radius), the constraint on central $q$ grew weaker, as expected. The limiting condition of specifying the elongation of the outermost flux surface, which is well determined by external magnetics, provides no information as to the value of $q_{0}$.

The sensitivity of this measurement was tested by supplying the elongation of a flux surface as a constraint in addition to external magnetic measurements. Using a Monte Carlo analysis, the starting parameters of the reconstruction were changed randomly and the code was allowed to converge to a solution. As is typical of many nonlinear fitting algorithms, the starting point of the reconstruction can have a significant effect on the final solution. If the collection of solutions from the Monte Carlo analysis has a consistent value of $q_{0}$, than the elongation measurement at that flux surface serves as a strong constraint.

When the elongation was specified at the 0.15 normalized flux surface (about 30\% horizontal extent in plasma minor radius), the Monte Carlo reconstructions gave an average value of $q_{0}=1.02$ with a standard deviation $\sigma=0.04$. Specifying the measurement at the 0.3 normalized flux surface resulted in an average value of $q_{0}=1.6$ with $\sigma=0.2$. Finally, at the 0.5 normalized flux surface, the Monte Carlo reconstructions gave $q_{0}=2.4$ and $\sigma=3.3$, thus illustrating the strong reduction in sensitivity as the shape measurement moves further from the magnetic axis.

The Monte Carlo method was also used to investigate the sensitivity of the $q$ profile to a tangential x-ray image projection. As the final solution approaches the expected solution of $q_{0}=1.2$, the summed value of the weighted residual between the measured and calculated tangential projection $\chi^{2}$ decreases significantly demonstrating a better fit to the measurements (Fig. 2). Mentioned previously, the final solution of a nonlinear fit can strongly depend on the initial parameters. Though the starting parameters for the Monte Carlo study have a large variation, in practice, starting parameters for the nonlinear fit will be chosen that closely resemble typical Pegasus plasma equilibriums which will result in more consistent solutions. 


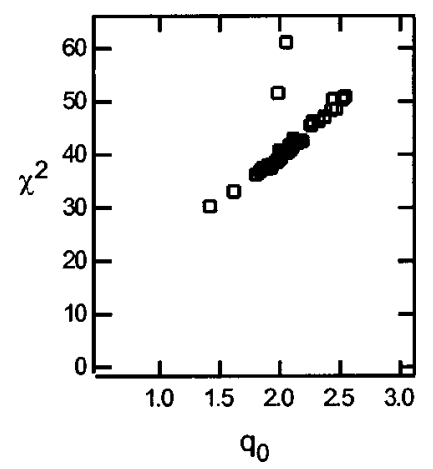

FIG. 2. Monte Carlo equilibrium reconstructions demonstrating sensitivity of nonlinear fitting routine to $2 \mathrm{D}$ tangential image constraint for determination of the central safety factor $q_{0}$.

\section{HARDWARE}

Initial tests were performed with a prototype PHC system. This system consisted of a $1 \mathrm{~mm}$ diam pinhole coupled to a $20 \mathrm{~cm}$ diam vacuum viewport. The viewport was coated with $\mathrm{Gd}_{2} \mathrm{O}_{2} \mathrm{~S}: \mathrm{Tb}(\mathrm{P} 43)$ phosphor and lens coupled to an image intensified charge coupled device (CCD) camera system. While the prototype did measure a tangential image, signal levels were low and long integration times $(>5 \mathrm{~ms})$ were necessary. The first generation PHC system was built based on the signal information gathered from the prototype.

It has been demonstrated that a significant gain $(\sim \times 5)$ in phosphor light output can be obtained by using a back reflective system. ${ }^{10}$ This method avoids optical attenuation which occurs when the phosphor emission travels through the bulk material. This increase combined with a larger diameter microchannel plate (MCP) reduced the photon noise of the imaging system. Additionally, a $\mathrm{Gd}_{2} \mathrm{O}_{2} \mathrm{~S}$ :Pr phosphor was used instead P43 as it has a much shorter afterglow period. ${ }^{11}$

The soft x-ray tangential PHC has a $4 \mathrm{~mm}$ diam, pinhole assembly coupled to a $30 \mathrm{~cm} \times 20 \mathrm{~cm}$ phosphor plate (Fig. $3)$. The pinhole assembly includes a variable filter cartridge to allow the selection of different filter materials and thicknesses. As stated previously, the reflective emission of the phosphor plate is then lens coupled to a $40 \mathrm{~mm}$ diam MCP detector. The MCP serves to boost the signal and also act as a high speed shutter allowing exposures from a few ms to $<100 \mu$ s. The MCP is lens coupled to a $512 \times 512$ Princeton Instruments (PI) 16 bit CCD camera. The PI camera uses water and Peltier cooling to achieve thermal stability and reduced dark noise. The camera has no exposure control and a slow readout, thus requiring active shuttering using the MCP.

The initial x-ray images from Pegasus included a high level of emission from the plasma edge. These low energy $x$ rays were generated from oxygen impurities in the scrape off layer and edge of the plasma. Using a thin beryllium filter $(0.2 \mu \mathrm{m})$, the higher energy emission from the core of the plasma was overwhelmed by this lower energy signal. Equilibrium reconstruction using these images was problematic due to the nonmonotonic nature of the $\mathrm{x}$-ray emission as a function of normalized flux. The thicker beryllium filters reduced the edge radiation, but attenuated the total x-ray signal to unusable levels.

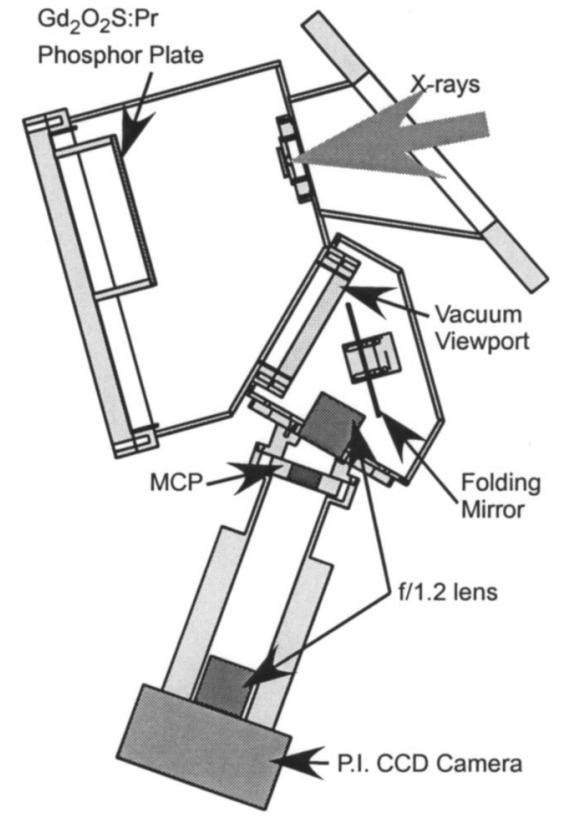

FIG. 3. Schematic of first generating soft x-ray pinhole camera on the Pegasus Toroidal experiment. Light from the phosphor is collected in the reflective mode; gated and intensified using a MCP.

To solve this problem, an $\mathrm{x}$-ray transmission filter code was developed which used the plasma x-ray emission modeling code combined with a nonlinear least squares fitting routine to maximize the ratio between the core and edge plasma emission while simultaneously maximizing the total signal level. Based on the standard assumptions for Pegasus plasma conditions, a thin nickel filter $(0.2 \mu \mathrm{m})$ was found to be a very good candidate for rejection of the low energy edge emission. The ideal filter was found to be a $0.2 \mu \mathrm{m} \mathrm{MgF}$ filter with a thin layer of $\mathrm{Al}$ for visible light opacity. The nickel filters were readily obtained and installed. The resultant images taken with the $0.2 \mu \mathrm{m}$ nickel filter in place demonstrate a strong contrast compared to the $0.2 \mu \mathrm{m}$ beryllium filter. While the signal level from the core of the plasma remains essentially the same, the pollution from the edge of the plasma is entirely eliminated (Fig. 4).

Present images have a lower signal level than expected based on calculations of the optical train throughtput and output from a plasma $\mathrm{x}$-ray modeling code. Other, solidstate, $\mathrm{x}$-ray devices demonstrate signal levels consistent with modeled x-ray emission. Also, initial calibration tests with a Manson x-ray source indicate that the phosphor and optical train throughput is comparable to calculated predictions. As the x-ray emission from Pegasus plasmas is dominated by oxygen impurity emission, there could be some question as to the efficiency of the phosphor as a function of energy. The phosphor may have a lower than expected conversion efficiency in the energy range of the primary emission from Pegasus. To resolve this issue, tests are currently underway to measure the conversion efficiency of the phosphor as a function of incident $\mathrm{x}$-ray energy.

While the noise of the present system is dominated by photon statistics at the MCP, the next generation PHC system will improve the signal to noise ratio through direct illumi- 

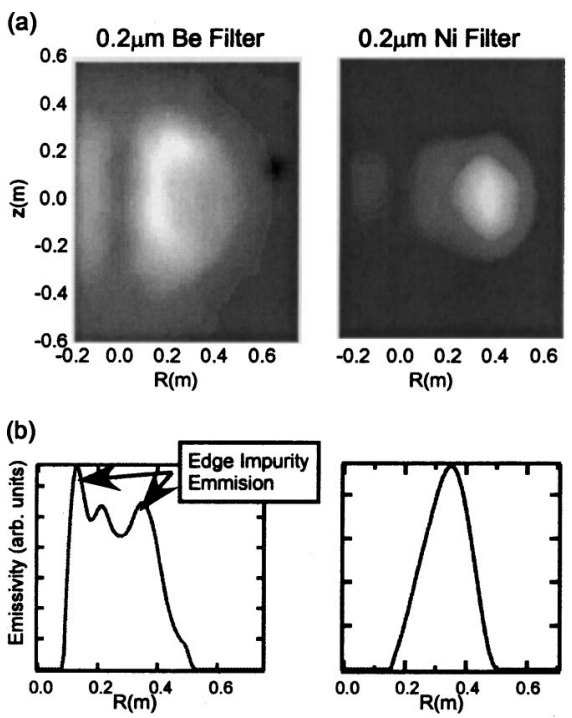

FIG. 4. (a) X-ray images from the PHC demonstrating the rejection of the low energy edge emission using the $\mathrm{Ni}$ filter instead of the Be filter. (b) corresponding Abel-inverted midplane emission plots.

nation of a CCD chip. This new system will use a small pinhole $(50 \mu \mathrm{m})$ directly coupled to a SiTE (Scientific Imaging Technologies, Inc.) 502 CCD chip mounted in a camera from Finger Lakes Instruments. The signal achieved with this new system is expected to be $50 \times$ higher than the previous system, as the silicon conversion efficiency is much greater than the product of the phosphor conversion efficiency, the MCP quantum efficiency, and the etendue of the lens coupling system.

The idea of a direct illumination system was initially discarded due to lack of availability of a high speed, low noise CCD camera system with a high dynamic range. A high readout speed was deemed necessary to prevent smearing of the image from lack of x-ray exposure control. However, a novel technique involving use of a custom designed $\mathrm{x}$-ray mask and CCD line transfer control allows exposure control and multiple time point capability by interleaving $\mathrm{x}$-ray images behind a tantalum mask. 32 evenly spaced lines of the CCD are exposed to the x-ray emission from the plasma. After each exposure, the horizontal rows of CCD pixels are shifted down, both to protect the previous exposure, and to allow the collection of the next time slice into the newly exposed row of pixels. Because the PHC system has a large angular field of view, the tantalum mask must be positioned to within a few tens of microns from the CCD chip to minimize shadowing and vignetting effects (Fig. 5).

\section{DISCUSSION}

Reconstruction modeling has demonstrated the effectiveness of using flux surface shape information as a constraint on the internal plasma profiles. The combination of external magnetics, which specify the boundary and outer region of the plasma, and a tangential x-ray image of the core of the plasma, which strongly constrains the interior current profile, allows a full plasma equilibrium reconstruction. In addition,
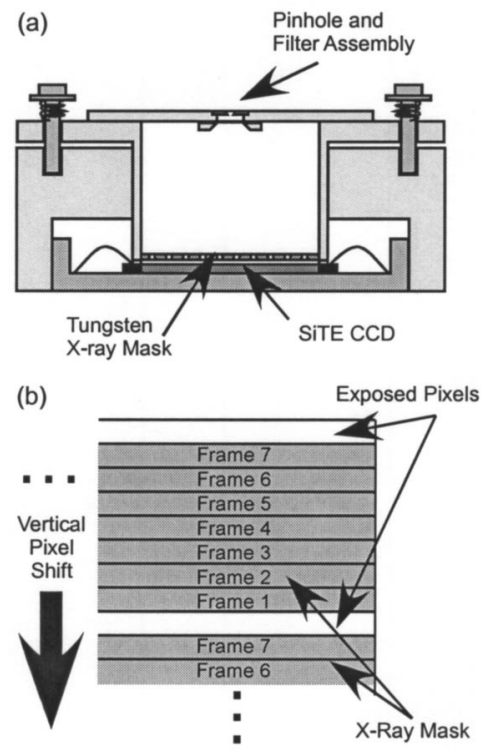

FIG. 5. (a) Schematic of direct illumination x-ray pinhole camera system. (b) Illustration of interleaving multiple frames and exposure control using vertical pixel shifting of CCD sensor and x-ray shielding with tantalum mask.

the simplicity and passive nature of the imaging system make the SXR PHC diagnostic attractive both for smaller experimental programs, and for larger programs that operate in regimes where traditional profile diagnostics become problematic.

The next generation direct exposure x-ray imaging system is currently under testing and construction and should be available for measurements once Pegasus resumes operation. The increased sensitivity and simplicity of design of the new system will improve the quality of image data and streamline the use of flux surface shape data as an equilibrium constraint for routine plasma reconstructions on the Pegasus Toroidal Experiment.

\section{ACKNOWLEDGMENT}

This work was supported by the U.S. DOE Grant No. DE-FG0296ER54375.

${ }^{1}$ G. D. Garstka et al., 29th EPS Conference on Plasma Physics and Controlled Fusion, Montreux, Canada, 17-21 June 2002.

${ }^{2}$ K. Tritz, R. Fonck, and T. Thorson, Rev. Sci. Instrum. 70, 595 (1999).

${ }^{3}$ J. P. Christiansen and J. B. Taylor, Nucl. Fusion 22, 111 (1982).

${ }^{4}$ M. C. Borrás and R. S. Granetz, Plasma Phys. Controlled Fusion 38, 289 (1996).

${ }^{5}$ J. P. Christiansen, J. D. Callen, J. J. Ellis, and R. S. Granetz, Nucl. Fusion 29, 703 (1989).

${ }^{6}$ E. T. Powell et al., Nucl. Fusion 33, 1493 (1993).

${ }^{7}$ A. Holland, R. J. Fonck, E. T. Powell, and S. Sesnic, Rev. Sci. Instrum. 59, 1819 (1988).

${ }^{8}$ M. E. Mauel et al., Plasma Phys. Controlled Nucl. Fusion Res. 1, 415 (1988).

${ }^{9}$ W. H. Press, S. A. Teukolsky, W. T. Vetterling, and B. P. Flannery, Numerical Recipes in $C$, 2nd ed. (Cambridge University Press, Cambridge, 1995).

${ }^{10}$ A. Baciero, L. Placentino, K. J. McCarthy, L. R. Barquero, A. Ibarra, and B. Zurro, J. Appl. Phys. 85, 6790 (1999).

${ }^{11}$ J. P. Creasey and G. C. Tyrrell, Proc. SPIE 3942, 114 (2000). 